Review

\title{
Proteins Directly Interacting with Mammalian 20S Proteasomal Subunits and Ubiquitin-Independent Proteasomal Degradation
}

\section{Raúl Sánchez-Lanzas ${ }^{1,2}$ and José G. Castaño ${ }^{1,2, *}$}

1 Departamento de Bioquímica, Instituto de Investigaciones Biomédicas 'Alberto Sols', UAM-CSIC; Facultad de Medicina de la Universidad Autónoma de Madrid, Madrid 28029, Spain;

E-Mail: rslanzas@iib.uam.es

2 Centro de Investigación Biomédica en Red sobre Enfermedades Neurodegenerativas (CIBERNED); Valderrebollo 5, Madrid 28041, Spain

* Author to whom correspondence should be addressed; E-Mail: joseg.castano@uam.es; Tel.: +34-91-497-5498; Fax: +34-91-585-4401.

Received: 1 June 2014; in revised form: 25 November 2014 / Accepted: 11 December 2014 / Published: 19 December 2014

\begin{abstract}
The mammalian 20S proteasome is a heterodimeric cylindrical complex ( $\alpha 7 \beta 7 \beta 7 \alpha 7$ ), composed of four rings each composed of seven different $\alpha$ or $\beta$ subunits with broad proteolytic activity. We review the mammalian proteins shown to directly interact with specific 20S proteasomal subunits and those subjected to ubiquitin-independent proteasomal degradation (UIPD). The published reports of proteins that interact with specific proteasomal subunits, and others found on interactome databases and those that are degraded by a UIPD mechanism, overlap by only a few protein members. Therefore, systematic studies of the specificity of the interactions, the elucidation of the protein regions implicated in the interactions (that may or may not be followed by degradation) and competition experiments between proteins known to interact with the same proteasomal subunit, are needed. Those studies should provide a coherent picture of the molecular mechanisms governing the interactions of cellular proteins with proteasomal subunits, and their relevance to cell proteostasis and cell functioning.
\end{abstract}

Keywords: proteasome; proteasome interactions; ubiquitin-dependent; ubiquitin-independent; degradation; proteolysis; proteasome activators; transcription; cell cycle; neurodegeneration 


\section{Introduction}

The proteasome is a $2.5 \mathrm{MDa}$ complex formed by a proteolytic core particle (20S, CP), and is a cylindrical shaped complex with a heterodimeric structure ( $\alpha 7 \beta 7 \beta 7 \alpha 7$ subunits). Attached to both bases of the cylinder is a regulatory particle (19S, RP) that consists of a horseshoe-like complex composed of a base and a lid. There are two copies of each of the three catalytic $\beta$ subunits $(\beta 1, \beta 2$ and $\beta 5)$ in the $\mathrm{CP}$ and their active sites are located inside the catalytic chamber formed by the contiguous $\beta$ rings [1].

The consensus mechanism of protein degradation by the $26 \mathrm{~S}$ proteasome states that ubiquitin must be attached to the protein so that it can be tagged for degradation. The process starts with the recognition of the poly-ubiquitylated protein which is carried out by the base of the RP which leads to de-ubiquitylation (the lid of the RP), the unfolding of the protein (the base), and translocation into the catalytic chamber of the CP for proteolysis [1]. However, an increasing number of studies have proved the existence of alternative mechanisms for protein degradation by the proteasome, which do not require prior ubiquitylation. Proteins directly degraded by a ubiquitin-independent proteasomal degradation mechanism (UIPD) must belong to the large set of proteins that interact with the proteasome which include modulators or accessory proteins of proteasomal function.

Our aim is to provide a critical assessment of the research carried out in this area, by analyzing the specific interactions of mammalian cellular proteins with specific 20S (CP) proteasomal subunits. This will enable identification of the set of proteins that interact with the proteasome, and a comparison of these proteins with the set of proteins degraded by the UIPD mechanism [2]. Finally, we provide some suggestions for further research in this area.

\section{Interaction of Cellular Proteins with Specific Proteasomal $\alpha$ and $\beta$ Subunits of the 20S Proteasome Complex}

We performed several general (alpha or beta proteasome subunits) or specific (using the acronym of each subunit) searches into the published literature to identify proteins interacting with specific CP subunits. Although we endeavoured to carry out as comprehensive a survey as possible, it may be possible that some papers have been overlooked, which we recognize poses some limitations to this work. A succinct description of those protein interacting partners of specific CP subunits, and the consequences of those interactions is given below.

\subsection{PSMA2, C3, $\alpha 2$}

The PSMA2 subunit of the $20 \mathrm{~S}$ proteasome complex has been shown to directly interact with $\mathrm{I \kappa B} \alpha$ through its arm-repeats [3] likely mediating its UIPD. More recently it has been shown that calcineurin also interacts with PSMA2 and promotes the degradation of $\mathrm{I} \kappa \mathrm{B} \alpha$ by the ubiquitin-proteasome pathway [4].

\subsection{PSMA4, C9, $\alpha 3$}

The PSMA4 subunit interacts with amino acids 40 to 60 of Hepatitis $\mathrm{C}$ virus F protein and promotes its UIPD [5]. 


\subsection{PSMA7, XAPC7, $\alpha 4$}

The PSMA7 subunit has been reported to be one of the $\alpha$-subunits that interacts with the REG $\alpha / \beta$ (PA28 $\alpha / \beta$ ) proteasomal activator as shown by yeast two-hybrid experiments, and the inhibition of proteasomal activation by the hepatitis $\mathrm{B}$ virus $\mathrm{X}$ protein-derived polypeptide, which binds directly to the PSMA7 subunit [6]. PSMA7 C-terminus also interacts specifically with the N-terminal region of Rab7 and participates in the late endocytic transport of cargo proteins, but this interaction does not promote Rab7 degradation [7]. Parkin, an E3 ligase implicated in Parkinson disease (PD), interacts through its C-terminus IBR-RING with the C-terminal region of PSMA7, and it may function as an accessory protein for substrate presentation to the proteasome for degradation [8]. The reported interaction of hypoxia-inducible factor-1 $\alpha$ (HIF-1 $\alpha)$ with PSMA7 [9] suggests that it regulates its degradation and is prevented by the direct interaction of PSMA7 with calcineurin B, this results in the inhibition of HIF-1 $\alpha$ degradation by the proteasomal pathway [10]. In the same context, Endothelial Monocyte Activating Polypeptide-II (EMAP-II) interacts with PSMA7 after internalization, increasing the degradation of HIF-1 $\alpha$ under hypoxic conditions [11]. Finally, PSMA7 also interacts with the nucleotide-binding oligomerization domain-containing protein 1 (NOD1) promoting its degradation by the proteasome [12].

\subsection{PSMA3, C8, $\alpha 7$ Subunit}

PSMA3 is known to form double ring heptameric structures (540 kDa) when expressed as a recombinant protein in bacteria [13]. PSMA3 is also able to form heterogeneous $540 \mathrm{kDa}$ complexes with alphaB-crystallin, although alphaB-crystallin does not directly interact with the proteasome [14]. PSMA3 is also one of the subunits that interacts with $\mathrm{REG} \alpha / \beta(\mathrm{PA} 28 \alpha / \beta)$ mediating proteasomal activation [6] together with PSMA1 [15] and PSMA7 (as described above).

Egr-1 [16] and aurora/Ip11-related kinase 2 (Aurora-B) [17] interact with PSMA3, but it is unclear if those interactions are involved in the ubiquitin-dependent proteasomal degradation (UDPD) of Egr-1 or Aurora-B. The C-terminus of p21WAF1/CIP1 interacts with PSMA3 promoting its degradation by a UIPD mechanism [18]. Apart from this direct interaction, several proteins have been shown to mediate presentation of $\mathrm{p} 21$ to the proteasome complex. MDM2, an E3 ubiquitin ligase, does not ubiquitylate p21, but through the region comprising amino acids 180-298, binds to $\mathrm{p} 21$, enhancing the binding of p21 to the PSMA3 proteasomal subunit for UIPD of p21 [19]. 14-3-3tau protein also binds to p21, MDM2, and PSMA3, facilitating the targeting of p21 to degradation [20]. Finally, binding p21 to REG $\gamma$ (PA28 $\gamma$ ) a proteasome activator, also seems to facilitate p21 degradation by the proteasome $[21,22]$. Id-1 interacts with PSMA3, and this interaction seems to be critical for the degradation of the Hepatitis-B virus (HBV)-encoded protein, HBX which requires ubiquitylation to be degraded by the proteasome [23]. SRC-3/AIB1 is a steroid receptor coactivator that can interact directly with PSMA3 subunit [24] or bind to REG $\gamma$ (PA28 $\gamma$ ) for presentation to the proteasome for degradation [25]. MDM2 also binds to PSMA3 and promotes Rb-PSMA3 interaction, leading to UIPD of Rb [26,27]. PSMA3 also interacts with the Epstein-Barr virus (EBV)-encoded nuclear proteins EBNA3A, EBNA3B and EBNA3C that are directly degraded in vitro by the proteasome [28]. In vitro studies have also shown that PSMA3 interacts with splicing factors and other proteins involved in RNA metabolism [29]. 
Finally, the N-terminal region (amino acids 1-60) of alpha-synuclein, a protein implicated in PD, interacts with the C-terminal region of PSMA3, which is essential for its degradation by the $20 \mathrm{~S}$ proteasome [30].

\subsection{PSMB6, $Y, \beta 1$}

PSMB6 has been shown to interact with Plasminogen Activator Inhibitor-2 (PAI-2) and this interaction may mediate its anti-apoptotic role [31]. PSMB6 has also been shown to bind directly to p27Kip1 promoting its direct degradation by the proteasome [32,33].

\subsection{PSMB1, C5, $\beta 6$}

The intracellular domain of TrkA has been reported to interact with several proteins, including the PSMB1 proteasomal subunit, while TrkB and TrkC do not. This TRkA interaction results in the phosphorylation of PSMB1, although with unknown consequences in proteasomal function [34].

\subsection{PSMB4, N3, $\beta 7$}

HTLV-I Tax has been shown to interact with PSMB4 and may contribute to the targeting of either p105 or p65, and $\mathrm{I} \kappa \mathrm{B} \alpha$ to the proteasome for processing or degradation, respectively [35]. Smad1 is targeted for degradation by the ubiquitin-dependent mechanism as well as by binding to PSMB4, and to ornithine decarboxylase antizyme (Az), likely to be degraded by a UIPD mechanism [36].

The subunit specific interactions described above are schematically summarized in Figure 1, although this figure does not include the interaction with the proteasomal activators REG $\alpha / \beta(\mathrm{PA} 28 \alpha / \beta)$ and REG $\gamma(\mathrm{PA} 28 \gamma)$.

Figure 1. Schematic diagram of $20 \mathrm{~S}$ proteasomal subunits and mammalian cellular proteins reported to interact with specific proteasomal subunits. The different $\alpha$ subunits are shaded in bright yellow and the $\beta$ subunits are pale yellow. Active $\beta$ subunits ( $\beta 1, \beta 2$ and $\beta 5$ ) are coloured black degraded to pale yellow. Mammalian proteins reported to interact with a specific proteasomal subunit are indicated by rectangular boxes. For further details refer to the main text.

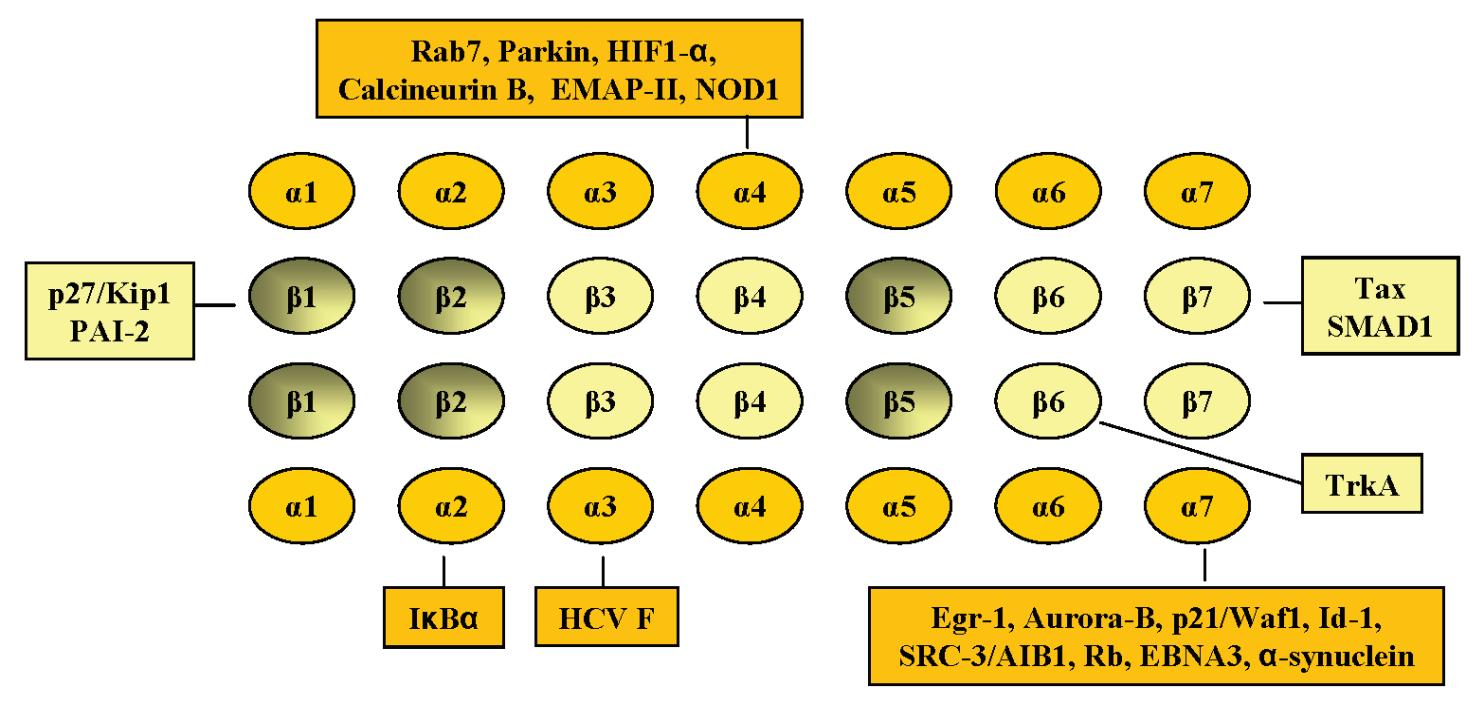




\section{Ubiquitin Independent Proteasomal Degradation}

The list of cellular proteins whose degradation does not necessitate prior ubiquitylation is increasing. The proteins that have already been described [2,37] include: ornithine decarboxylase (ODC) either directly or mediated by the Az, p21 (see above), p53 whose degradation is inhibited by NAD(P)H:quinone oxidoreductase 1 (NQO1), c-Fos also inhibited by NQO1 [38] and Fra-1 which interacts with the19S proteasomal subunit, TBP-1 that has a TBP-1 ubiquitin-independent C-terminal degron [39], Rb presented by human cytomegalovirus pp71 protein or MDM2 (see above), alpha-synuclein (see above), HIF-1 $\alpha$, SRC-3/AIB1 transcriptional coactivator (see above), NF- $\kappa$ B p105 processing into p50 and the degradation of I $\mathrm{B} \alpha$ (see above), Y-box-binding protein 1 (YB-1), thymidylate synthase (TS) and Tau protein which is implicated in Alzheimer's disease.

Further proteins have been added to the list of those being degraded by a UIPD mechanism since the last extensive revision [2]. The new proteins reported to be degraded (presented below) are ordered by the date of publication, and include proteins implicated in many cellular functions or pathways.

Proteins implicated in DNA and chromatin structure degraded by UIPD include BAF57, a component of the mammalian SWI/SNF chromatin remodelling complex [40], and Topoisomerase II $\beta$ (Top2 $\beta$ ) degraded by the $26 \mathrm{~S}$ proteasome after RNA polymerase II blockage [41]. Transcription factors reported to be degraded by a UIDP mechanism include: KLF5 a Kruppel-like zinc finger transcription factor [42], DNp73, a transactivation-deficient and anti-apoptotic form of p73 whose degradation is mediated by $\mathrm{Az}$ [43], Bob1 (Obf-1 or OCA-B) a transcriptional coactivator [44] and IאBNS which acts as an inhibitor of a subset of NF- $\kappa$ B target genes [45].

Proteins implicated in cell cycle control and apoptosis that have been reported to be degraded by UIPD include: Aurora-A mediated by Az and regulated by binding to AURKAIP1 [46], Daxx whose degradation is promoted by human cytomegalovirus (HCMV) pp71 [47], MCL-1 anti-apoptotic myeloid cell leukemia 1 [48], BIM-extra long (BIM(EL) a pro-apoptotic BH3-only protein [49], NOXA an unstructured BH3-only protein [50], a homeodomain transcription factor NKX3.1 whose degradation is mediated by its C-terminal 21-amino acid domain [51], and nucleostemin a nucleolar GTP-binding protein essential for ribosomal biogenesis whose degradation is controlled by GTP levels [52].

Viral proteins degraded by UIPD include: HBX which probably regulates gene transcription [53], human cytomegalovirus (HCMV) pUL21 a protein required for establishing an HCMV infection [54], murine cytomegalovirus (MCMV) pM141 a protein that together with pM140 is required for virion assembly [55] and the hepatitis $\mathrm{C}$ virus (HCV) p7 protein, a hexameric protein forming a funnel-like structure in the membranes which play a critical role in the virion life-cycle [56].

Other proteins with diverse cellular functions reported to be degraded by UIPD include: processing of the N-terminus of LC3 an ubiquitin-like protein that plays an essential role in autophagy [57], connexin43 (Cx43) whose degradation is stimulated by CIP75 [58], RILaltCterm an alternatively spliced isoform of RIL that activates actin bundling [59], voltage-gated Kv7.2/KCNQ2/M-channel C-terminal which has a frame-shift mutation that has been found in benign familiar epilepsy [60], DJ-1 L166P a missense mutant implicated in familiar forms of PD [61] and RCHY1 whose degradation is mediated by interaction with Hoxa2 [62].

We have also added to this list those proteins that become substrates for UIPD via their interaction with proteasomal activators, mainly REG $\gamma$ (PA28 $\gamma$ ) and PA200/B1m10. We have already described that 
REG $\gamma$ (PA28 $\gamma$ ) can present p21, but it can also present other cell cycle regulators such as p16 (INK4A) and p19 (Arf) to proteasomes for degradation [21,22] and SRC-3 a coactivator for UIPD [25]. The levels of activation-induced deaminase (AID), responsible for the initiation of antibody gene diversification

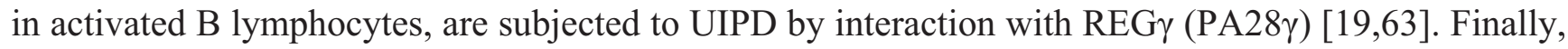
MAFA, a basic leucine zipper transcription factor implicated in insulin gene transcriptional regulation, interacts with REG $\gamma$ (PA28 $\gamma$ ) for proteasomal degradation; this interaction is dependent on MAFA phosphorylation by GSK-3 [64]. REG $\gamma$ (PA28 $\gamma$ ) also seems also to facilitate the interaction of p53 and MDM2, but in this case it promotes MDM2-dependent UDPD of p53 [65] and participates in the mechanism of the regulation of HCV core proteins, nuclear retention and degradation [66,67]. The PA200/Blm10 proteasome activator binds to the CP by its C-terminal YYX motif and activates in vitro degradation of tau [68] which is known to be mediated by the 20S proteasome [69]. More recently it has been shown that PA200/Blm10 promotes the UIPD of acetylated core histones by binding to the bromodomain-like regions of PA200 [70].

The proteins shown to be degraded by a UIPD mechanism are summarized in Figure 2, they have been classified according to the function or the cellular process in which they are involved. Many of the proteins involved in transcription, cell cycle and apoptosis, and which are also degraded by a UDPD mechanism, appear prominently as UIPD substrates. However, this fact may only be a reflection of the active research bias in these areas as demonstrated by the large number of PubMed entries retrieved using those keywords in a search.

Figure 2. Classification of mammalian proteins reported to be degraded by an ubiquitin-independent proteasomal pathway. The protein function or cellular processes in which those proteins are involved have been used to group the different mammalian proteins that have been shown to be subjected to Ubiquitin-Independent Proteasomal Degradations (UIPD) by the $20 \mathrm{~S}$ or $26 \mathrm{~S}$ proteasome, or facilitated by activators (PA28 $\gamma$ and PA200) of the proteasome. A full description and details of the individual proteins and their UIPD mechanism may be found in the main text.

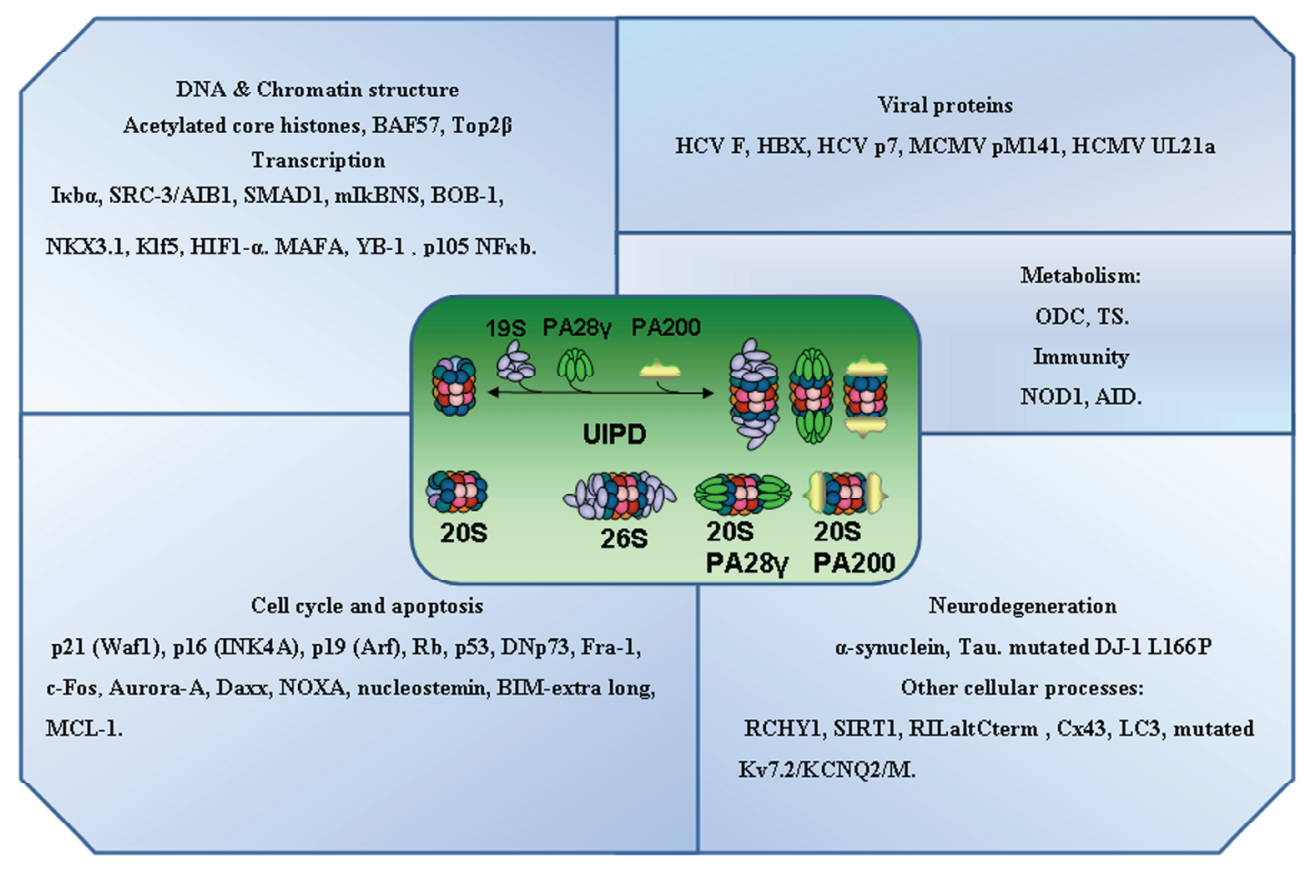




\section{A Critical Assessment of Specific Protein Interactions of Proteasomal Subunits and UIPD}

Proteins shown to interact directly with the different mammalian $20 \mathrm{~S}$ proteasomal subunits and which do not belong to the RP complex are summarized in Figure 1. Those proteins constitute a set which are not all degraded by a UIPD mechanism (Figure 2). Many more proteasomal interacting proteins can be found in interactome databases. For example, PSMA3 in the BIOGRID interactome database is reported to interact with 148 different cellular proteins without taking into account those protein partners that are components of the proteasomal CP or RP. The protein-partners of PSMA3 shown in Figure 1 are included in interactomic databases. In general, the high-throughput methods generate interactome data sets that still have numerous false positives; therefore further experimental evidence is needed to ascertain the relevance of those interactions.

Proteasome-like structures are as old in evolutionary terms, as C-terminal diglycine ubiquitin or ubiquitin-like molecules, and are unevenly distributed in the different kingdoms including Archea [71]. If the unique function of the simple Archea proteasome, formed by only one (or two) type(s) of $\alpha$ and $\beta$ subunits, was protein degradation, this would suggest the existence of an archaic macromolecular complex with hidden catalytic sites which would offer two types of subunits for interactions with "thousands" of protein substrates for degradation or other functional consequences. What is the molecular basis of those interactions? Certainly, our knowledge is very limited at present. Duplication of the $\alpha$ and $\beta$ genes, and diversification of their sequences has occurred during evolution [71]. In order to maintain the functional structure of the proteasome, those sequence changes must occur in regions that are not relevant, or that are compensated by changes in the corresponding interacting subunits of the proteasome complex. This would be necessary to keep the basic cylindrical structure and the correct processing of the active pre- $\beta$ subunits. In eukaryotes, which have fourteen types of CP proteasomal subunits, the number of cellular protein interacting partners is likely to have increased. What are the specific sequences and structural determinants responsible for the presumed increase in the number of interacting proteins? The answer may be provided by studies of the proteins that interact with archeal proteasomal subunits together with high-resolution X-ray data of this proteasome and the proteasome-cellular protein complexes (which would probably also be feasible for yeast proteasomes). The results of these investigations may eventually provide the atomic details of the binding site locations and the physicochemical properties of the interaction interfaces. These studies could also provide us with an evolutionary perspective, and would certainly help discover the basic 'highly' conserved principles of the interactions between cellular proteins and proteasomal subunits, as well as providing a better understanding of the UIPD mechanism.

To validate a direct interaction between a specific proteasomal subunit and a specific cellular protein, most of the published research relies on two-hybrid studies and affinity-capture followed by mass spectrometry. Another experimental approach is the immunoblotting of pull-down experiments of crude cellular extracts, cell-free translation products or purified recombinant proteins using antibodies or recombinant proteins. Specificity, when analyzed, is defined by the use of proteasomal subunits that do not interact with the protein under study, or with a structural modification of the proteasomal subunit and/or the corresponding interacting protein partner. Those structure variants allow the determination of which regions of both partners are involved in the interaction. Alternatively, some groups have used cells expressing tagged proteasomal subunits to explore the binding of endogenous, or transfected tagged, cellular protein partners by co-immunoprecipitation or binding to affinity-resins. 
A critical analysis of the in vitro experiments reveals that the reported interactions that either cannot be reproduced using the entire proteasomal complex (because the structure and the surface offered by the proteasomal subunits in the complex differs from the unassembled proteasomal subunits) or, where the interaction cannot be demonstrated in cell lysates, are irrelevant. This criticism would be correct for those interacting protein partners that are not directly degraded by the proteasome, but would not be sustainable for those that are degraded by the proteasome. In this case, the productive interaction ends in an enzymatic reaction. Accordingly, it may be difficult to isolate the CP-interacting protein intermediate of the reaction even at lower temperatures, or in the presence of proteasome inhibitors. The binding energy may contribute to lowering the energy barrier of peptide bond hydrolysis by an induced conformational change of the proteasome and/or the active sites. The alternative to validate the reported interaction would be through kinetic competition experiments, using specific inhibition of the degradation of the interacting protein by constructs of the specific proteasomal subunit in vitro or by transfection in cells, provided that it is demonstrated that the proteasome complex structure is not affected. Furthermore, competition experiments using proteins that are reported to bind to the same (or different, allowing specificity of the competition to be studied) proteasomal subunits would be very helpful to understand the relative kinetic constants and the strength of the interactions between the different proteins.

The same experimental approach could also be applied to cell studies albeit with some obvious limitations. One clear limitation to the analysis of the interactions with proteasome subunits in the cell would be that the amount of free and unassembled $20 \mathrm{~S}$ proteasomal subunits is likely to be very low, with the possible exception of tumor cells where they are overproduced and degraded [72,73]. Competition experiments by over expression of a proteasomal subunit (untagged or tagged in the C-terminus) will displace the corresponding endogenous subunit and assemble instead in the newly synthesized and assembled proteasome. Unless the transfected proteasomal subunit remains unassembled in the cell, it would be difficult to validate these experiments as proof of the specific interaction of a protein with a specific proteasomal subunit. The same applies to the interruption of the expression of proteasomal subunits by sh or siRNA interference. The time required to downregulate the quantity of one or several subunits from the 'old, fully assembled' and pre-existing 20S complex is dependent on the half-life of the mature 20S complex, which is estimated to be more than a week [74]. In view of these caveats, one clear way to demonstrate the relevance in vivo of a specific interaction found in vitro would be to demonstrate the competition between two protein partners that bind to the same proteasomal subunit.

The number of proteins which participate in many cellular functions (Figure 2), described as being degraded by a UIPD mechanism is clearly increasing. It has been estimated that approximately $20 \%$ of total cell protein could be degraded by a UIPD mechanism [75]. The criteria used to establish that a protein is degraded by this mechanism are: the Lys-less version of the protein substrate (all Lys mutated to Arg) must be degraded by the proteasome, and blocking the N-terminal Met either chemically or with a tag, should not affect the degradation of the Lys-less protein by the proteasome. The above criteria exclude both internal Lys and N-terminal Met ubiquitylation establishing a UIPD mechanism for that particular protein. An exception would be those proteins that may not require Lys or Met for ubiquitin conjugation and degradation, but N-terminal acetylation [76,77] by a UIPD mechanism. To our knowledge, no such proteins have been reported. Not all the proteins reported as degraded by a UIPD mechanism (described above and summarized in Figure 2) fully satisfy the criteria mentioned above. Furthermore, many of those reported to be degraded by UIPD can also be degraded by a UDPD 
mechanism. The relevance or the significance of the existence of two mechanisms (UIPD and UDPD) for the same cellular protein is unclear. We could speculate that UIPD may be a default proteostatic mechanism, while UDPD a fast-adaptive response to control the proteostasis of those proteins.

There have been some attempts to determine the minimal requirements of a protein substrate to be degraded by a UIPD mechanism [78-80], but its generalization is unclear. The consensus is that many of the proteins degraded by UIPD mechanism have in toto (p21, $\alpha$-synuclein, Tau) or in part (p53, HIF1- $\alpha$ ) of its sequence, a so-called unstructured region [81,82]. Energetically, it can be conceived that those regions will facilitate their binding and translocation in the interior of the catalytic chamber of the proteasome. However, the specific and non-specific interactions with proteasomal subunits that mediate the process need to be defined. A critical issue is the determination of which of the proteasomal subunits, of both the $20 \mathrm{~S}$ or $19 \mathrm{~S}$ complexes, specifically interact with those proteins reported to be degraded by a UIPD mechanism.

Finally, another criticism commonly made in the reports of the UIPD of a protein substrate is that the 19S complex or proteasomal activators have to participate in this process. This is because the $\alpha$-ring channel of the CP is too narrow and has to be opened to allow the transit of the extended protein into the catalytic chamber where it will be degraded [1]. As a consequence of this principle, the CP alone is inactive, except for small peptides that may diffuse freely. It is not easy to answer the question about whether the $20 \mathrm{~S}$ proteasome has proteolyitc activity or only peptidase activity. Probably, only NMR studies will be able to unambiguously answer that question. In the meantime, it would be useful to think that proteasomes may not only behave as top-down degrading nano-cylinders (the predominant vision nowadays), but may also be lateral degrading nano-cylinders, like a lawn-mower (Figure 2, central diagram). Broadening the paradigm, protein substrates may also access the catalytic chamber of the CP through the space between the $\alpha$ and $\beta$ rings of the cylinder. This heterodox hypothesis has already been postulated when the crystal structure of the yeast 20S proteasome was reported [83].

\section{Conclusions}

Proteasome subunits have been shown to interact with many cellular proteins; we have only described those, reported in specific published research papers, which interact with mammalian CP subunits. It is time to start to qualify the specificity, the regions of both partners implicated in the process, and to quantify, using competition experiments, the relative strength of the interactions of those proteins, both in vitro and in vivo. The degradation of cellular proteins by a UIPD mechanism implies the binding, either directly or indirectly (via another protein interacting with the proteasome) of those proteins to proteasomal subunits. It is also time to determine the proteasomal subunits responsible for this UIPD mechanism. Again, specificity, the protein regions implicated, and competition experiments will contribute to a better understanding of the mechanism of their proteasomal degradation. Both approaches could combine to form a coherent picture of the relevance of the cellular protein interactions with the proteasome and proteasomal function (including the UIDP mechanism) in cell proteostasis and cell function. Further investigations of the molecular basis of the UIDP and UDPD mechanism of the same proteins, where applicable, and those that are only degraded by a UDPD mechanism, would provide a relational and hierarchical view of the proteasome pathway, as the main proteostatic mechanism of protein degradation in the cell. 


\section{Acknowledgments}

We thank Alejandra C. Abrusci for English correction. This work was supported by grants from MINECO SAF-2012-34556, Comunidad de Madrid P2010_BMD-2331 \& CIBERNED to José G. Castaño.

\section{Conflicts of Interest}

The authors declare no conflict of interest.

\section{References}

1. Tomko, R.J., Jr.; Hochstrasser, M. Molecular architecture and assembly of the eukaryotic proteasome. Annu. Rev. Biochem. 2013, 82, 415-445.

2. Jariel-Encontre, I.; Bossis, G.; Piechaczyk, M. Ubiquitin-independent degradation of proteins by the proteasome. Biochim. Biophys. Acta 2008, 1786, 153-177.

3. Alvarez-Castelao, B.; Castano, J.G. Mechanism of direct degradation of IkappaBalpha by $20 \mathrm{~S}$ proteasome. FEBS Lett. 2005, 579, 4797-4802.

4. Zhang, W.; Wei, Q. Calcineurin stimulates the expression of inflammatory factors in RAW 264.7 cells by interacting with proteasome subunit alpha type 6. Biochem. Biophys. Res. Commun. 2011, 407, 668-673.

5. Yuksek, K.; Chen, W.L.; Chien, D.; Ou, J.H. Ubiquitin-independent degradation of hepatitis C virus F protein. J. Virol. 2009, 83, 612-621.

6. Stohwasser, R.; Holzhutter, H.G.; Lehmann, U.; Henklein, P.; Kloetzel, P.M. Hepatitis B virus HBx peptide 116-138 and proteasome activator PA28 compete for binding to the proteasome alpha4/MC6 subunit. Biol. Chem. 2003, 384, 39-49.

7. Dong, J.; Chen, W.; Welford, A.; Wandinger-Ness, A. The proteasome alpha-subunit XAPC7 interacts specifically with Rab7 and late endosomes. J. Biol. Chem. 2004, 279, 21334-21342.

8. Dachsel, J.C.; Lucking, C.B.; Deeg, S.; Schultz, E.; Lalowski, M.; Casademunt, E.; Corti, O.; Hampe, C.; Patenge, N.; Vaupel, K.; et al. Parkin interacts with the proteasome subunit alpha4. FEBS Lett. 2005, 579, 3913-3919.

9. Cho, S.; Choi, Y.J.; Kim, J.M.; Jeong, S.T.; Kim, J.H.; Kim, S.H.; Ryu, S.E. Binding and regulation of HIF-1alpha by a subunit of the proteasome complex, PSMA7. FEBS Lett. 2001, 498, 62-66.

10. Li, N.; Zhang, Z.; Zhang, W.; Wei, Q. Calcineurin B subunit interacts with proteasome subunit alpha type 7 and represses hypoxia-inducible factor-1alpha activity via the proteasome pathway. Biochem. Biophys. Res. Commun. 2011, 405, 468-472.

11. Tandle, A.T.; Calvani, M.; Uranchimeg, B.; Zahavi, D.; Melillo, G.; Libutti, S.K. Endothelial monocyte activating polypeptide-II modulates endothelial cell responses by degrading hypoxia-inducible factor-1alpha through interaction with PSMA7, a component of the proteasome. Exp. Cell Res. 2009, 315, 1850-1859.

12. Yang, L.; Tang, Z.; Zhang, H.; Kou, W.; Lu, Z.; Li, X.; Li, Q.; Miao, Z. PSMA7 directly interacts with NOD1 and regulates its function. Cell. Physiol. Biochem. 2013, 31, 952-959. 
13. Gerards, W.L.; Enzlin, J.; Haner, M.; Hendriks, I.L.; Aebi, U.; Bloemendal, H.; Boelens, W. The human alpha-type proteasomal subunit $\mathrm{HsC} 8$ forms a double ringlike structure, but does not assemble into proteasome-like particles with the beta-type subunits HsDelta or HsBPROS26. J. Biol. Chem. 1997, 272, 10080-10086.

14. Boelens, W.C.; Croes, Y.; de Jong, W.W. Interaction between alphaB-crystallin and the human 20S proteasomal subunit C8/alpha7. Biochim. Biophys. Acta 2001, 1544, 311-319.

15. Kania, M.A.; DeMartino, G.N.; Baumeister, W.; Goldberg, A.L. The proteasome subunit, C2, contains an important site for binding of the PA28 (11S) activator. Eur. J. Biochem. 1996, 236, 510-516.

16. Bae, M.H.; Jeong, C.H.; Kim, S.H.; Bae, M.K.; Jeong, J.W.; Ahn, M.Y.; Bae, S.K.; Kim, N.D.; Kim, C.W.; Kim, K.R.; et al. Regulation of Egr-1 by association with the proteasome component C8. Biochim. Biophys. Acta 2002, 1592, 163-167.

17. Shu, F.; Guo, S.; Dang, Y.; Qi, M.; Zhou, G.; Guo, Z.; Zhang, Y.; Wu, C.; Zhao, S.; Yu, L. Human aurora-B binds to a proteasome alpha-subunit $\mathrm{HC} 8$ and undergoes degradation in a proteasome-dependent manner. Mol. Cell. Biochem. 2003, 254, 157-162.

18. Touitou, R.; Richardson, J.; Bose, S.; Nakanishi, M.; Rivett, J.; Allday, M.J. A degradation signal located in the C-terminus of $\mathrm{p} 21 \mathrm{WAF} 1 / \mathrm{CIP} 1$ is a binding site for the $\mathrm{C} 8$ alpha-subunit of the $20 \mathrm{~S}$ proteasome. EMBO J. 2001, 20, 2367-2375.

19. Zhang, Z.; Wang, H.; Li, M.; Agrawal, S.; Chen, X.; Zhang, R. MDM2 is a negative regulator of p21WAF1/CIP1, independent of p53. J. Biol. Chem. 2004, 279, 16000-16006.

20. Wang, B.; Liu, K.; Lin, H.Y.; Bellam, N.; Ling, S.; Lin, W.C. 14-3-3Tau regulates ubiquitin-independent proteasomal degradation of $\mathrm{p} 21$, a novel mechanism of $\mathrm{p} 21$ downregulation in breast cancer. Mol. Cell. Biol. 2010, 30, 1508-1527.

21. Chen, X.; Barton, L.F.; Chi, Y.; Clurman, B.E.; Roberts, J.M. Ubiquitin-independent degradation of cell-cycle inhibitors by the REG $\gamma$ proteasome. Mol. Cell 2007, 26, 843-852.

22. Li, X.; Amazit, L.; Long, W.; Lonard, D.M.; Monaco, J.J.; O’Malley, B.W. Ubiquitin- and ATP-independent proteolytic turnover of p21 by the REG $\gamma$-proteasome pathway. Mol. Cell 2007, 26, 831-842.

23. Ling, M.T.; Chiu, Y.T.; Lee, T.K.; Leung, S.C.; Fung, M.K.; Wang, X.; Wong, K.F.; Wong, Y.C. Id-1 induces proteasome-dependent degradation of the HBX protein. J. Mol. Biol. 2008, 382, 34-43.

24. Yi, P.; Feng, Q.; Amazit, L.; Lonard, D.M.; Tsai, S.Y.; Tsai, M.J.; O’Malley, B.W. Atypical protein kinase $\mathrm{C}$ regulates dual pathways for degradation of the oncogenic coactivator SRC-3/AIB1. Mol. Cell 2008, 29, 465-476.

25. Li, X.; Lonard, D.M.; Jung, S.Y.; Malovannaya, A.; Feng, Q.; Qin, J.; Tsai, S.Y.; Tsai, M.J.; O'Malley, B.W. The SRC-3/AIB1 coactivator is degraded in a ubiquitin- and ATP-independent manner by the REG $\gamma$ proteasome. Cell 2006, 124, 381-392.

26. Sdek, P.; Ying, H.; Chang, D.L.; Qiu, W.; Zheng, H.; Touitou, R.; Allday, M.J.; Xiao, Z.X. MDM2 promotes proteasome-dependent ubiquitin-independent degradation of retinoblastoma protein. Mol. Cell 2005, 20, 699-708.

27. Ying, H.; Xiao, Z.X. Targeting retinoblastoma protein for degradation by proteasomes. Cell Cycle 2006, 5, 506-508. 
28. Touitou, R.; O'Nions, J.; Heaney, J.; Allday, M.J. Epstein-Barr virus EBNA3 proteins bind to the C8/alpha7 subunit of the $20 \mathrm{~S}$ proteasome and are degraded by $20 \mathrm{~S}$ proteasomes in vitro, but are very stable in latently infected B cells. J. Gen. Virol. 2005, 86, 1269-1277.

29. Fedorova, O.A.; Moiseeva, T.N.; Nikiforov, A.A.; Tsimokha, A.S.; Livinskaya, V.A.; Hodson, M.; Bottrill, A.; Evteeva, I.N.; Ermolayeva, J.B.; Kuznetzova, I.M.; et al. Proteomic analysis of the 20S proteasome (PSMA3)-interacting proteins reveals a functional link between the proteasome and mRNA metabolism. Biochem. Biophys. Res. Commun. 2011, 416, 258-265.

30. Alvarez-Castelao, B.; Goethals, M.; Vandekerckhove, J.; Castano, J.G. Mechanism of cleavage of alpha-synuclein by the $20 \mathrm{~S}$ proteasome and modulation of its degradation by the RedOx state of the N-terminal methionines. Biochim. Biophys. Acta 2014, 1843, 352-365.

31. Fan, J.; Zhang, Y.Q.; Li, P.; Hou, M.; Tan, L.; Wang, X.; Zhu, Y.S. Interaction of plasminogen activator inhibitor-2 and proteasome subunit, beta type 1. Acta Biochim. Biophys. Sin. 2004, 36, 42-46.

32. Tambyrajah, W.S.; Bowler, L.D.; Medina-Palazon, C.; Sinclair, A.J. Cell cycle-dependent caspase-like activity that cleaves $\mathrm{p} 27^{\mathrm{KIP} 1}$ is the $\beta_{1}$ subunit of the $20 \mathrm{~S}$ proteasome. Arch. Biochem. Biophys. 2007, 466, 186-193.

33. Yuan, F.; Ma, Y.; You, P.; Lin, W.; Lu, H.; Yu, Y.; Wang, X.; Jiang, J.; Yang, P.; Ma, Q.; et al. A novel role of proteasomal betal subunit in tumorigenesis. Biosci. Rep. 2013, 33, 555-565.

34. MacDonald, J.I.; Verdi, J.M.; Meakin, S.O. Activity-dependent interaction of the intracellular domain of rat Trka with intermediate filament proteins, the $\beta-6$ proteasomal subunit, Ras-GRF1, and the p162 subunit of eIF3. J. Mol. Neurosci. 1999, 13, 141-158.

35. Beraud, C.; Greene, W.C. Interaction of HTLV-I Tax with the human proteasome: Implications for NF-кB induction. J. Acquir. Immune Defic. Syndr. Hum. Retrovirol. 1996, 13, S76-S84.

36. Gruendler, C.; Lin, Y.; Farley, J.; Wang, T. Proteasomal degradation of Smad1 induced by bone morphogenetic proteins. J. Biol. Chem. 2001, 276, 46533-46543.

37. Erales, J.; Coffino, P. Ubiquitin-independent proteasomal degradation. Biochim. Biophys. Acta 2014, 1843, 216-221.

38. Adler, J.; Reuven, N.; Kahana, C.; Shaul, Y. c-Fos proteasomal degradation is activated by a default mechanism, and its regulation by $\mathrm{NAD}(\mathrm{P}) \mathrm{H}$ :quinone oxidoreductase 1 determines c-Fos serum response kinetics. Mol. Cell Biol. 2010, 30, 3767-3778.

39. Pakay, J.L.; Diesch, J.; Gilan, O.; Yip, Y.Y.; Sayan, E.; Kolch, W.; Mariadason, J.M.; Hannan, R.D.; Tulchinsky, E.; Dhillon, A.S. A 19S proteasomal subunit cooperates with an ERK MAPK-regulated degron to regulate accumulation of Fra-1 in tumour cells. Oncogene 2012, 31, 1817-1824.

40. Keppler, B.R.; Archer, T.K. Ubiquitin-dependent and ubiquitin-independent control of subunit stoichiometry in the SWI/SNF complex. J. Biol. Chem. 2010, 285, 35665-35674.

41. Ban, Y.; Ho, C.W.; Lin, R.K.; Lyu, Y.L.; Liu, L.F. Activation of a novel ubiquitin-independent proteasome pathway when RNA polymerase II encounters a protein roadblock. Mol. Cell Biol. 2013, 33, 4008-4016.

42. Chen, C.; Zhou, Z.; Guo, P.; Dong, J.T. Proteasomal degradation of the KLF5 transcription factor through a ubiquitin-independent pathway. FEBS Lett. 2007, 581, 1124-1130. 
43. Dulloo, I.; Gopalan, G.; Melino, G.; Sabapathy, K. The antiapoptotic DeltaNp73 is degraded in a c-Jun-dependent manner upon genotoxic stress through the antizyme-mediated pathway. Proc. Natl. Acad. Sci. USA 2010, 107, 4902-4907.

44. Lindner, J.M.; Wong, C.S.; Moller, A.; Nielsen, P.J. A C-terminal acidic domain regulates degradation of the transcriptional coactivator Bob1. Mol. Cell Biol. 2013, 33, 4628-4640.

45. Park, K.C.; Jeong, J.; Kim, K.I. Regulation of mIkappaBNS stability through PEST-mediated degradation by proteasome. Biochem. Biophys. Res. Commun. 2014, 443, 1291-1295.

46. Lim, S.K.; Gopalan, G. Antizyme1 mediates AURKAIP1-dependent degradation of Aurora-A. Oncogene 2007, 26, 6593-6603.

47. Hwang, J.; Kalejta, R.F. Proteasome-dependent, ubiquitin-independent degradation of Daxx by the viral pp71 protein in human cytomegalovirus-infected cells. Virology 2007, 367, 334-338.

48. Stewart, D.P.; Koss, B.; Bathina, M.; Perciavalle, R.M.; Bisanz, K.; Opferman, J.T. Ubiquitin-independent degradation of antiapoptotic MCL-1. Mol. Cell Biol. 2010, 30, 3099-3110.

49. Wiggins, C.M.; Tsvetkov, P.; Johnson, M.; Joyce, C.L.; Lamb, C.A.; Bryant, N.J.; Komander, D.; Shaul, Y.; Cook, S.J. BIM(EL), an intrinsically disordered protein, is degraded by $20 \mathrm{~S}$ proteasomes in the absence of poly-ubiquitylation. J. Cell Sci. 2011, 124, 969-977.

50. Craxton, A.; Butterworth, M.; Harper, N.; Fairall, L.; Schwabe, J.; Ciechanover, A.; Cohen, G.M. NOXA, a sensor of proteasome integrity, is degraded by $26 \mathrm{~S}$ proteasomes by an ubiquitin-independent pathway that is blocked by MCL-1. Cell Death Differ. 2012, 19, 1424-1434.

51. Rao, V.; Guan, B.; Mutton, L.N.; Bieberich, C.J. Proline-mediated proteasomal degradation of the prostate-specific tumor suppressor NKX3.1. J. Biol. Chem. 2012, 287, 36331-36340.

52. Lo, D.; Dai, M.S.; Sun, X.X.; Zeng, S.X.; Lu, H. Ubiquitin- and MDM2 E3 ligase-independent proteasomal turnover of nucleostemin in response to GTP depletion. J. Biol. Chem. 2012, 287, 10013-10020.

53. Kim, J.H.; Sohn, S.Y.; Benedict Yen, T.S.; Ahn, B.Y. Ubiquitin-dependent and -independent proteasomal degradation of hepatitis B virus X protein. Biochem. Biophys. Res. Commun. 2008, 366, 1036-1042.

54. Fehr, A.R.; Yu, D. Human cytomegalovirus gene UL21a encodes a short-lived cytoplasmic protein and facilitates virus replication in fibroblasts. J. Virol. 2010, 84, 291-302.

55. Bolin, L.L.; Hanson, L.K.; Slater, J.S.; Kerry, J.A.; Campbell, A.E. Murine cytomegalovirus US22 protein pM140 protects its binding partner, pM141, from proteasome-dependent but ubiquitin-independent degradation. J. Virol. 2010, 84, 2164-2168.

56. Haqshenas, G. The $\mathrm{p} 7$ protein of hepatitis $\mathrm{C}$ virus is degraded via the proteasome-dependent pathway. Virus Res. 2013, 176, 211-215.

57. Gao, Z.; Gammoh, N.; Wong, P.M.; Erdjument-Bromage, H.; Tempst, P.; Jiang, X. Processing of autophagic protein LC3 by the 20S proteasome. Autophagy 2010, 6, 126-137.

58. Su, V.; Nakagawa, R.; Koval, M.; Lau, A.F. Ubiquitin-independent proteasomal degradation of endoplasmic reticulum-localized connexin43 mediated by CIP75. J. Biol. Chem. 2010, 285, 40979-40990.

59. Guryanova, O.A.; Drazba, J.A.; Frolova, E.I.; Chumakov, P.M. Actin cytoskeleton remodeling by the alternatively spliced isoform of PDLIM4/RIL protein. J. Biol. Chem. 2011, 286, 26849-26859. 
60. Su, J.; Cao, X.; Wang, K. A novel degradation signal derived from distal C-terminal frameshift mutations of KCNQ2 protein which cause neonatal epilepsy. J. Biol. Chem. 2011, 286, 42949-42958.

61. Alvarez-Castelao, B.; Munoz, C.; Sanchez, I.; Goethals, M.; Vandekerckhove, J.; Castano, J.G. Reduced protein stability of human DJ-1/PARK7 L166P, linked to autosomal recessive Parkinson disease, is due to direct endoproteolytic cleavage by the proteasome. Biochim. Biophys. Acta 2012, 1823, 524-533.

62. Bergiers, I.; Bridoux, L.; Nguyen, N.; Twizere, J.C.; Rezsohazy, R. The homeodomain transcription factor Hoxa2 interacts with and promotes the proteasomal degradation of the E3 ubiquitin protein ligase RCHY1. PLoS One 2013, 8, e80387.

63. Uchimura, Y.; Barton, L.F.; Rada, C.; Neuberger, M.S. REG-gamma associates with and modulates the abundance of nuclear activation-induced deaminase. J. Exp. Med. 2011, 208, 2385-2391.

64. Kanai, K.; Aramata, S.; Katakami, S.; Yasuda, K.; Kataoka, K. Proteasome activator PA28 $\gamma$ stimulates degradation of GSK3-phosphorylated insulin transcription activator MAFA. J. Mol. Endocrinol. 2011, 47, 119-127.

65. Zhang, Z.; Zhang, R. Proteasome activator PA28 $\gamma$ regulates p53 by enhancing its MDM2-mediated degradation. EMBO J. 2008, 27, 852-864.

66. Moriishi, K.; Okabayashi, T.; Nakai, K.; Moriya, K.; Koike, K.; Murata, S.; Chiba, T.; Tanaka, K.; Suzuki, R.; Suzuki, T.; et al. Proteasome activator PA28y-dependent nuclear retention and degradation of hepatitis C virus core protein. J. Virol. 2003, 77, 10237-10249.

67. Suzuki, R.; Moriishi, K.; Fukuda, K.; Shirakura, M.; Ishii, K.; Shoji, I.; Wakita, T.; Miyamura, T.; Matsuura, Y.; Suzuki, T. Proteasomal turnover of hepatitis C virus core protein is regulated by two distinct mechanisms: A ubiquitin-dependent mechanism and a ubiquitin-independent but PA28 $\gamma$-dependent mechanism. J. Virol. 2009, 83, 2389-2392.

68. Dange, T.; Smith, D.; Noy, T.; Rommel, P.C.; Jurzitza, L.; Cordero, R.J.; Legendre, A.; Finley, D.; Goldberg, A.L.; Schmidt, M. Blm10 protein promotes proteasomal substrate turnover by an active gating mechanism. J. Biol. Chem. 2011, 286, 42830-42839.

69. David, D.C.; Layfield, R.; Serpell, L.; Narain, Y.; Goedert, M.; Spillantini, M.G. Proteasomal degradation of tau protein. J. Neurochem. 2002, 83, 176-185.

70. Qian, M.X.; Pang, Y.; Liu, C.H.; Haratake, K.; Du, B.Y.; Ji, D.Y.; Wang, G.F.; Zhu, Q.Q.; Song, W.; $\mathrm{Yu}, \mathrm{Y}$.; et al. Acetylation-mediated proteasomal degradation of core histones during DNA repair and spermatogenesis. Cell 2013, 153, 1012-1024.

71. Gille, C.; Goede, A.; Schloetelburg, C.; Preissner, R.; Kloetzel, P.M.; Gobel, U.B.; Frommel, C. A comprehensive view on proteasomal sequences: Implications for the evolution of the proteasome. J. Mol. Biol. 2003, 326, 1437-1448.

72. Kumatori, A.; Tanaka, K.; Inamura, N.; Sone, S.; Ogura, T.; Matsumoto, T.; Tachikawa, T.; Shin, S.; Ichihara, A. Abnormally high expression of proteasomes in human leukemic cells. Proc. Natl. Acad. Sci. USA 1990, 87, 7071-7075.

73. Nandi, D.; Woodward, E.; Ginsburg, D.B.; Monaco, J.J. Intermediates in the formation of mouse $20 \mathrm{~S}$ proteasomes: Implications for the assembly of precursor beta subunits. EMBO J. 1997, 16, 5363-5375.

74. Cuervo, A.M.; Palmer, A.; Rivett, A.J.; Knecht, E. Degradation of proteasomes by lysosomes in rat liver. Eur. J. Biochem. 1995, 227, 792-800. 
75. Borris, T.J.; Gebhart, D.L. An unusual case of an erupting microsupernumerary tooth. Gen. Dent. 1991, 39, 286-287.

76. Hwang, C.S.; Shemorry, A.; Varshavsky, A. N-terminal acetylation of cellular proteins creates specific degradation signals. Science 2010, 327, 973-977.

77. Kim, H.K.; Kim, R.R.; Oh, J.H.; Cho, H.; Varshavsky, A.; Hwang, C.S. The N-terminal methionine of cellular proteins as a degradation signal. Cell 2014, 156, 158-169.

78. Melo, S.P.; Barbour, K.W.; Berger, F.G. Cooperation between an intrinsically disordered region and a helical segment is required for ubiquitin-independent degradation by the proteasome. J. Biol. Chem. 2011, 286, 36559-36567.

79. Singh Gautam, A.K.; Balakrishnan, S.; Venkatraman, P. Direct ubiquitin independent recognition and degradation of a folded protein by the eukaryotic proteasomes-origin of intrinsic degradation signals. PLoS One 2012, 7, e34864.

80. Alfassy, O.S.; Cohen, I.; Reiss, Y.; Tirosh, B.; Ravid, T. Placing a disrupted degradation motif at the $\mathrm{C}$ terminus of proteasome substrates attenuates degradation without impairing ubiquitylation. J. Biol. Chem. 2013, 288, 12645-12653.

81. Dyson, H.J.; Wright, P.E. Intrinsically unstructured proteins and their functions. Nat. Rev. Mol. Cell Biol. 2005, 6, 197-208.

82. Suskiewicz, M.J.; Sussman, J.L.; Silman, I.; Shaul, Y. Context-dependent resistance to proteolysis of intrinsically disordered proteins. Protein Sci. 2011, 20, 1285-1297.

83. Groll, M.; Ditzel, L.; Lowe, J.; Stock, D.; Bochtler, M.; Bartunik, H.D.; Huber, R. Structure of 20S proteasome from yeast at $2.4 \AA$ resolution. Nature $1997,386,463-471$.

(C) 2014 by the authors; licensee MDPI, Basel, Switzerland. This article is an open access article distributed under the terms and conditions of the Creative Commons Attribution license (http://creativecommons.org/licenses/by/4.0/). 